\title{
Suppression of Apoptosis in the Liver by Systemic and Local Delivery of Small-Interfering RNAs
}

\author{
Lars Zender and Stefan Kubicka
}

\begin{abstract}
Summary
RNA interference (RNAi) is a sequence-specific gene-silencing mechanism triggered by doublestranded RNA. RNAi was shown to allow transient or stable knockdown of gene expression in a broad range of species and has been used successfully for functional genomic screens in mammalian cells and Caenorhabditis elegans. Standard therapeutic use of RNAi in clinical settings in humans has been hampered by the lack of effective methods to deliver the small-interfering RNAs (siRNAs) or short-hairpin RNA expression vectors into the diseased organs. In mice, systemic delivery of siRNAs by hydrodynamic intravascular injection leads to highly efficient uptake of siRNAs into the liver. Several groups demonstrated therapeutic use of RNAi in mouse models of acute liver failure or hepatitis B virus replication. This chapter will focus on the technical background of hydrodynamic and portal vein delivery techniques in mice and will give practical guidance for using these techniques for siRNA delivery into the liver.
\end{abstract}

Key Words: siRNA; RNA interference; hydrodynamic intravascular injection; apoptosis; acute liver failure.

\section{Introduction}

RNA interference (RNAi) has been shown to be a powerful tool to study gene function in a broad range of different organisms. It overcomes the limitations of classical genetic methods to knockdown gene expression in mammalian cells. Most recently, RNAi has been exploited as a highthroughput genomics tool in mammals (1-3). Furthermore, RNAi holds great promise as a therapeutic tool. Applications of RNAi directed against targets in diseases, for example cancer, infectious diseases, or dominant genetic diseases, are conceivable. Although the therapeutic use in humans is

\footnotetext{
From: Methods in Molecular Biology, vol. 361, Target Discovery and Validation Reviews and Protocols Volume 2, Emerging Molecular Targets and Treatment Options Edited by: M. Sioud (c) Humana Press Inc., Totowa, NJ
} 
still some time from becoming routine in the clinic, use of experimental delivery methods in mice currently allows the study of RNAi in preclinical therapeutic settings (4-7).

Because numerous comprehensive reviews about RNAi are available in the literature, this chapter will not give a detailed background on the discovery of RNAi but will focus on practical aspects of using RNAi in mice.

\subsection{Efficient Delivery: the Holy Grail of Gene Therapeutic Approaches}

The holy grail of all in vivo gene therapeutic approaches is to deliver effective amounts of the therapeutic gene into the desired target cells. Currently, viral vectors are most widely used for delivery of therapeutic genes in vivo. However, vector-associated side effects limit their value as a tool for clinical use.

Systemic application of plasmid DNA or antisense oligonucleotides (ASO), however, leads to very low uptake in most tissues. In part, this is owing to the fact that nucleic acids are rapidly degraded after systemic intravascular application in vivo. With regard to future therapeutic applications in humans, it is promising that under certain conditions short-interfering RNA (siRNA) duplexes seem to be more stable than chemically modified ASO (8). Several approaches have been described to achieve more efficient uptake of naked DNA or ASO into tissues in vivo. Among these are direct injection into the target organ, as shown for muscle and liver $(9,10)$ and electroporation $(11,12)$.

\subsection{Hydrodynamic Intravascular Injection}

The large volume hydrodynamic delivery technique was a major advance in the in vivo delivery of nucleic acids into mice and rats. In principle, it means the injection of a high volume into the vascular system within a short period of time. The first report that this delivery method can be even more effective than direct injection into the target organ is from Budker et al. (13), who injected high volumes of either lacZ or luciferase reporter plasmids into the portal vein of mice. Using this approach approx $1 \%$ of all hepatocytes throughout the whole liver showed uptake of the plasmid. Remarkably, reporter activity was undetectable in endothelial and bile duct cells.

Because surgery on mice and intraportal injection is not feasible in every laboratory, it was an important discovery that injection of a high volume of plasmid DNA into the tail vein also leads to a very efficient uptake of DNA into the liver (14). In 2002, McCaffrey et al. showed that the hydrodynamic tail-vein delivery technique could be used to effectively deliver siRNA into hepatocytes (15). Subsequent studies showed that the siRNA uptake into the liver after hydrodynamic tail-vein delivery is much more effective than the 
uptake of plasmid DNA using this technique; uptake of siRNA in up to 70-90\% of all hepatocytes was achieved $(16,17)$.

The physiological mechanisms of the hydrodynamic intravascular injection technique are still not fully elucidated. Intravascular delivery of a large bolus of fluid is thought to result in an accumulation of the liquid in the caval vein. As the heart is swamped with the high amount of liquid, a temporary right heart failure with subsequent backflow into the liver is the consequence. In the liver, the fluid is thought to be extravasated through the pores (fenestrate) in the liver endothelium (sinusoids). It is well known that liver endothelium has a much bigger average pore size than endothelium of other organs. The observation that siRNA uptake into the liver after hydrodynamic tail-vein injection is much higher than the uptake of plasmid DNA is in line with the idea that extravasation through the endothelial pores is a prerequisite for uptake into hepatocytes. After passing the endothelium, the siRNA molecules or plasmid DNA are in direct contact with the hepatocytes. Whether the molecules are finally internalized by receptor mediated (18) or receptor independent processes like membrane rupture (19) remains unclear.

\subsection{Therapeutical Use of siRNA to Prevent and Treat Acute Liver Failure in Mice}

As the first demonstrations of a therapeutic application of siRNA in vivo were done in mouse models of acute liver failure (ALF), the next paragraphs will give a brief introduction into this disease, followed by a discussion of the therapeutic use of siRNA to suppress apoptosis in this model.

ALF is defined as a dramatic clinical syndrome in which a previously normal liver fails within days or weeks. Three subgroups of ALF can be distinguished, hyperacute, acute, and subacute liver failure. Despite the frequent occurrence of cerebral edema and renal failure in patients with hyperacute liver failure, prognosis without transplantation is relatively good. Survival rates in patients with acute and subacute liver failure, however, are at best $15 \%(20,21)$. The etiology of ALF shows marked worldwide variation: in underdeveloped countries viral causes predominate, whereas drug-induced hepatotoxicity and seronegative hepatitis predominate in most countries of the Western world (22). To this day, the management of these varying clinical scenarios is essentially supportive. It aims to identify and remove the insult that led to destruction of the liver, whereas preventing associated complications, such as acute renal and respiratory failure, bleeding diatheses, severe sepsis, cerebral edema, and encephalopathy. Overall mortality in patients with severe ALF remains high, ranging from 40 to $80 \%$. Although liver support devices or hepatocyte transplantation may in time have a place in treatment, currently liver transplantation remains the only therapeutic option that has 
been shown to significantly improve the outcome of patients with ALF. Because of limitations of donor organs and the requirement of lifelong and life-limiting immunosuppression, liver transplantation should be only performed in patients who are unlikely to recover from ALF. In patients who recover from ALF with medical support, the liver almost always returns to normal, both structurally and functionally. Consequently, prevention of destruction of liver cells in the time-course of ALF and support of liver regeneration are the most important goals in management of ALF by molecular therapies in the future.

Several molecular mechanisms can initiate liver cell injury and can further aggravate ongoing damage processes (23). Mitochondria are the prominent targets for hepatotoxicity of many drugs, leading to impairment of energy metabolism and intracellular oxidative stress. Once hepatocellular function is impaired, accumulation of hydrophobic bile acids causes additional cytotoxicity. Although drug-induced hepatotoxicity appears to be mediated by both apoptosis and necrosis, viral infection predominantly induces cell death of hepatocytes by apoptosis. In contrast to necrosis, apoptosis is a highly conserved physiological process important in normal development and tissue homeostasis of multicellular organisms. Apoptosis occurs by two pathways: a death receptor pathway and a mitochondrial pathway. Signals released from the cytoplasm and/or from the cell membrane activate a well-characterized cascade of caspases (cysteine aspartase), which execute apoptotic cell death (24-27). Receptor-mediated apoptosis, as triggered by the tumor necrosis factor-R, Fas-, or TNF-related apoptosis-inducing ligand (TRAIL)-receptor 1 , has been reported to be involved in the pathogenesis of different liver diseases like viral hepatitis, ALF, autoimmune hepatitis, ischemia-reperfusion injury, nonalcoholic steatohepatitis, and toxic liver damage like Wilson's disease or bile acid-induced hepatotoxicity (28-33). Therefore, the apoptotic pathway provides attractive targets for molecular therapy to prevent further liver damage and provides a condition for successful liver regeneration in ALF.

Recently, Song et al. (16) and our group (34) reported the therapeutic use of siRNA in mouse models of ALF. Song et al. used siRNA duplexes targeting the Fas (CD95) receptor. Three consecutive applications of Fas-siRNA led to an uptake of siRNA in more than $80 \%$ of all hepatocytes resulting in an 8- to 10-fold downregulation of Fas mRNA expression in the liver. To note, comparable downregulation of Fas had been shown before using ASO (35), however, this required treating the mice with an approx 14-fold higher amount of antiFas ASO (6 mg/kg body weight for 12 consecutive days). In accordance with the results from Zhang et al. (35), inhibition of Fas expression by siRNA protected the hepatocytes against treatment with the Fas-activating antibody Jo-2 
and resulted in significantly increased survival. Remarkably, Fas-siRNA also conferred protection against ConA-mediated acute liver damage, whereas Fasantisense did not.

As it is well established that in addition to FasL, tumor necrosis factor $\alpha$ and TRAIL are also involved in the pathogenesis of viral hepatitis $(36,37)$, we reasoned that an essential early downstream mediator of all death receptors would be the most suitable target to achieve the best therapeutic effects in preclinical animal models of viral hepatitis and ALF. To test this, we directed siRNA against caspase-8, which is a key downstream effector in receptor-mediated apoptosis. A single dose of $0.45-0.6 \mathrm{nmol} / \mathrm{g}$ body weight of caspase-8-siRNA resulted in very effective inhibition of caspase-8 expression in the liver, thus leading to protection against Jo-2-mediated liver damage or liver damage induced by an adenovirus overexpressing Fas ligand (Ad-FasL). With regard to potential clinical applications, it is noteworthy that caspase-8-siRNA not only prevented acute liver damage but was also highly effective when delivered into an ongoing ALF. Furthermore, it is of particular interest that in our study the therapeutic efficiency of caspase-8siRNA was shown in acute viral hepatitis that was triggered by wild-type adenovirus, which better resembles the multiple molecular events in human acute hepatitis.

\section{Materials}

\subsection{Preparation for Hydrodynamic Tail-Vein Injection in Mice}

1. Restraining device for mice.

2. Paper towels, warm water, or alternatively heating lamp.

3. 3-mL Syringe with screw thread, 21- to 27-gauge needle.

4. $0.9 \%$ Saline or Ringer's solution.

5. siRNA duplexes $(0.5-1.0 \mathrm{nmol} / \mathrm{g}$ body weight $)$.

\subsection{Preparation for Portal Vein Injection in Mice}

1. Surgery pad for mice.

2. Antiseptic solution.

3. 24-Gauge iv catheter.

4. Gauze.

5. Surgical instruments (scissors, tweezers, and retractors).

6. Ketamine/xylazine, alternatively gas-anesthsia (e.g., isoflurane may be used).

7. Suture material, 4-0.

8. Optional Fibrine/Thrombine adhesive solution (see Subheading 3.).

9. $0.9 \%$ Saline or Ringer's solution.

10. Lipiodol.

11. Microvascular clamps (optional). 
12. Adapter tube and infusion pump (optional).

13. siRNA duplex $(0.5-1.0 \mathrm{nmol} / \mathrm{g}$ body weight).

\section{Methods}

\subsection{Hydrodynamic/High Volume Tail-Vein Injection in Mice (see Note 1)}

In principle, any mouse strain can be used for this technique. BALB/c or other strains with white fur are preferred as visualization and puncture of the tail vein, especially with bigger needles, is more difficult in black mice.

We prefer to perform standard hydrodynamic tail-vein injection without anesthesia, as the combination of the high-volume injection together with anesthesia can lead to complications in some mice. If anesthesia is used, a gentle gas-anesthesia (e.g., isoflurane) should be preferred.

First, the injection solution is prepared. In our hands, best results are obtained with desalted, lyophilized siRNA duplexes, as siRNA duplexes lyophilized in annealing buffer can result in higher morbidity of the mice. Most of the "siRNA-companies" provide siRNA duplexes in a desalted "ready-to-go" option. The desired amount of siRNA duplex is dissolved in $0.9 \%$ saline or Ringer's solution. Effective siRNAs should work in a dose of $0.5-1.0 \mathrm{nmol}$ per gram body weight. The total injection volume can be calculated by dividing the mouse body weight by 10 (see Note 2 ).

The mouse is restrained using a suitable restraining device (numerous Plexiglas versions are available from different manufacturers) and a tail vein is dilated by application of warm water. Alternatively, some researchers dilate the tail vessels by placing the whole mouse under a heating lamp for $10 \mathrm{~min}$. A 3-mL syringe with a screw thread is connected to a needle. Needles from 21- to 27-gauges are suitable. Using syringes without a screw thread can lead to disconnection during hydrodynamic injection. A tail vein is punctured approximately midway between the tail tip and middle of the tail. If the first puncture is not successful, a more proximal puncture site can be tried. After injecting a small test volume to ensure that the needle is safely placed in the vein, the whole volume is applied within 4-10 s. Longer injection times will lead to less effective uptake of siRNA into the liver. Some mice will show heavy breathing and reduced activity after injection. These symptoms should not last longer than $30 \mathrm{~min}$.

\subsection{Intraportal Delivery of siRNA in Mice}

The hydrodynamic tail-vein injection technique leads to high and reproducible siRNA uptake into the liver. It offers the possibility to investigate the effects of siRNA-mediated gene knockdown in the "target organ" liver in 
different physiological and pathophysiological settings (see Note 3). However, a criticism against hydrodynamic tail-vein injection is that it is an experimental procedure that is not feasible in the clinical situation in humans. Therefore, it is desirable to have an experimental setting in the mouse model that can resemble the clinical situation in humans.

In humans the portal vein can be reached without open surgery by puncturing the jugular vein and placing a catheter from the inferior caval vein through the liver. Thus, the direct portal vein injection in mice can serve as a model for this procedure.

Animals are anesthetized with ketamine/Xxlazine or, if preferred, with gasanesthesia (e.g., isoflurane). The abdominal wall is cleaned with antiseptic solution. A midline ventral incision is made and the abdominal cavity is kept open with retractors. The bowels are wrapped in saline soaked gauze and placed sideward of the operation field to obtain a good view on the portal vein. The desired amount of siRNA $(0.5-1.0 \mathrm{nmol})$ is dissolved in saline or Ringer's solution containing $10 \%$ lipiodol as an embolizing agent. The use of an embolizing agent leads to a temporary stasis of the siRNA solution in the liver and, therefore, enhances the siRNA uptake into the liver. Alternatively, some researchers place microvascular clamps on the suprahepatic inferior caval vein during the injection procedure. This likewise prevents a fast flow-through of the siRNA solution through the liver. The portal vein is punctured with a 24-gauge iv catheter. After the plastic catheter is placed in a safe position the needle is removed. To avoid accidental movement of the catheter during injection, the portal vein is looped with a 4-0 ligature to tighten the catheter. A total volume of up to $5 \%$ of mouse body weight can be injected within $10 \mathrm{~s}$. Inexperienced researchers may find it easier to use an adapter tube to connect the catheter to an infusion-pump instead of injecting free hand. Before removing the needle, an adhesive solution of Fibrine/Thrombine is spread on the puncture site to prevent bleeding. The abdominal cavity is closed with two separate layers of 4-0 suture material (see Note 4). Fig. 1 shows the inhibition of endogenous expression of $L a c Z$ gene by hydrodynamic-derived siRNAs.

\section{Notes}

1. Researchers who are inexperienced with hydrodynamic tail-vein injection in mice may want to test their technical proficiency by using transgenic mice carrying a reporter gene like $l a c Z$ or green fluorescent protein (GFP). Using this approach with established siRNA duplexes against lacZ or GFP, the efficiency of siRNA delivery into the liver can be quantified (see also Fig. 1). Alternatively, Cy-5labeled siRNAs can be injected. An easy and precise way to quantify the percentage of liver cells with siRNA uptake is to prepare a single-cell suspension of liver cells using standard collagenase perfusion (38) and subsequently determine the Cy-5-positive fraction of hepatocytes by flow cytometry. In this context it is 
siRNA-scrambled

siRNA-lacZ

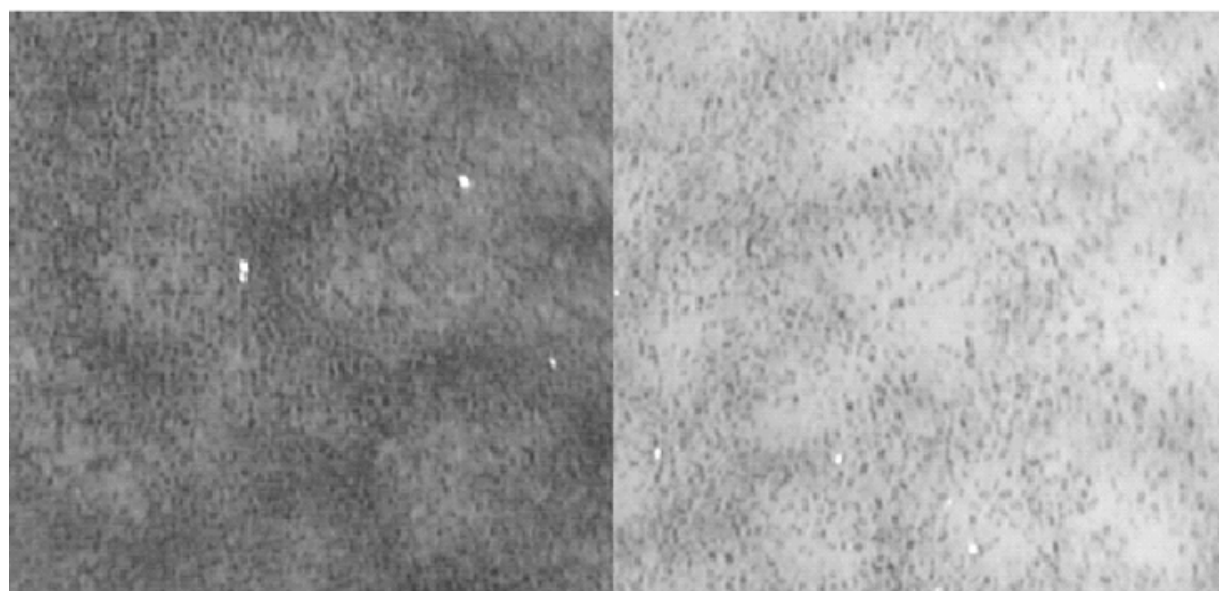

Fig. 1. Hydrodynamic tail-vein injection leads to efficient delivery of short-interfering RNAs (siRNA)-lacZ into the liver of lacZ-transgenic mice. Shown are photographs of the liver surface of lacZ-transgenic mice (C57BL/6J-TgN [MtnlacZ]) after hydrodynamic tail-vein injection of either siRNA-scrambled or siRNA-lacZ. LacZ staining was performed on whole liver (see Note 5).

important to mention that some siRNA duplexes lose their functionality when they are Cy-5-labeled.

2. To rule out off-target effects of siRNA, two independent siRNAs against every target should be used.

3. Although siRNA duplexes are effectively delivered to a target organ, the efficiency of knockdown of a specific gene must be quantified by assaying mRNA and protein expression.

4. Researchers who want to use the hydrodynamic tail-vein injection technique to deliver plasmid DNA to the liver are advised to work with supercoiled plasmid DNA that can be obtained easily by cesium chloride DNA preparations.

5. For experiments that need sustained knockdown of the target gene in the mouse liver, stability of the respective siRNA in the liver must be tested individually. Depending on the siRNA duplex, the duration of in vivo knockdown can be variable ranging from $2 \mathrm{~d}$ to more than $1 \mathrm{wk}$.

\section{Acknowledgments}

The authors would like to thank Drs. Mona S. Spector and Jesus Gil for critical reading of the manuscript.

\section{References}

1. Paddison, P. J., Silva, J. M., Conklin, D. S., et al. (2004) A resource for large-scale RNA-interference-based screens in mammals. Nature 428, 427-431. 
2. Berns, K., Hijmans, E. M., Mullenders, J., et al. (2004) A large-scale RNAi screen in human cells identifies new components of the p53 pathway. Nature 428, 431-437.

3. Brummelkamp, T. R., Nijman, S. M., Dirac, A. M., and Bernards, R. (2003) Loss of the cylindromatosis tumour suppressor inhibits apoptosis by activating NFkappaB. Nature 424, 797-801.

4. Zender, L., Hutker, S., Liedtke, C., et al. (2003) Caspase 8 small interfering RNA prevents acute liver failure in mice. Proc. Natl. Acad. Sci. USA 100, 7797-7802.

5. Song, E., Lee, S. K., Wang, J., et al. (2003) RNA interference targeting Fas protects mice from fulminant hepatitis. Nat. Med. 9, 347-351.

6. Klein, C., Bock, C. T., Wedemeyer, H., et al. (2003) Inhibition of hepatitis B virus replication in vivo by nucleoside analogues and siRNA. Gastroenterology 125, 9-18.

7. McCaffrey, A. P., Nakai, H., Pandey, K., et al. (2003) Inhibition of hepatitis B virus in mice by RNA interference. Nat. Biotechnol. 21, 639-644.

8. Bertrand, J. R., Pottier, M., Vekris, A., Opolon, P., Maksimenko, A., and Malvy, C. (2002) Comparison of antisense oligonucleotides and siRNAs in cell culture and in vivo. Biochem. Biophys. Res. Commun. 296, 1000-1004.

9. Danko, I., Williams, P., Herweijer, H., et al. (1997) High expression of naked plasmid DNA in muscles of young rodents. Hum. Mol. Genet. 6, 1435-1443.

10. Hickman, M. A., Malone, R. W., Lehmann-Bruinsma, K., et al. (1994) Gene expression following direct injection of DNA into liver. Hum. Gene Ther. 5, 1477-1483.

11. Hartikka, J., Sukhu, L., Buchner, C., et al. (2001) Electroporation-facilitated delivery of plasmid DNA in skeletal muscle: plasmid dependence of muscle damage and effect of poloxamer 188. Mol. Ther. 4, 407-415.

12. Somiari, S., Glasspool-Malone, J., Drabick, J. J., et al. (2000) Theory and in vivo application of electroporative gene delivery. Mol. Ther. 2, 178-187.

13. Budker, V., Zhang, G., Knechtle, S., and Wolff, J. A. (1996) Naked DNA delivered intraportally expresses efficiently in hepatocytes. Gene Ther. 3, 593-598.

14. Zhang, G., Budker, V., and Wolff, J. A. (1999) High levels of foreign gene expression in hepatocytes after tail vein injections of naked plasmid DNA. Hum. Gene Ther. 10, 1735-1737.

15. McCaffrey, A. P., Meuse, L., Pham, T. T., Conklin, D. S., Hannon, G. J., and Kay, M. A. (2002) RNA interference in adult mice. Nature 418, 38-39.

16. Song, E., Lee, S. K., Wang, J., et al. (2003) RNA interference targeting Fas protects mice from fulminant hepatitis. Nat. Med. 9, 347-351.

17. Zender, L., Hutker, S., Liedtke, C., et al. (2003) Caspase 8 small interfering RNA prevents acute liver failure in mice. Proc. Natl. Acad. Sci. USA 100, 7797-7802.

18. Budker, V., Budker, T., Zhang, G., Subbotin, V., Loomis, A., and Wolff, J. A. (2000) Hypothesis: naked plasmid DNA is taken up by cells in vivo by a receptormediated process. J. Gene Med. 2, 76-88.

19. Kobayashi, N., Kuramoto, T., Yamaoka, K., Hashida, M., and Takakura, Y. (2001) Hepatic uptake and gene expression mechanisms following intravenous administration of plasmid DNA by conventional and hydrodynamics-based procedures, J. Pharmacol. Exp. Ther. 297, 853-860. 
20. O'Grady, J. G., Schalm, S. W., and Williams, R. (1993) Acute liver failure: redefining the syndromes. Lancet 342, 273-275.

21. Plevris, J. N., Schina, M., and Hayes, P. C. (1998) Review article: the management of acute liver failure. Aliment. Pharmacol. Ther. 12, 405-418.

22. Bernal, W. (2003) Changing patterns of causation and the use of transplantation in the United Kingdom. Semin. Liver Dis. 23, 227-237.

23. Jaeschke, H., Gores, G. J., Cederbaum, A. I., Hinson, J. A., Pessayre, D., and Lemasters, J. J. (2002) Mechanisms of hepatotoxicity. Toxicol. Sci. 65, 166-176.

24. Adams, J. M. and Cory, S. (1998) The Bcl-2 protein family: arbiters of cell survival. Science 281, 1322-1326.

25. Ashkenazi, A. and Dixit, V. M. (1998) Death receptors: signaling and modulation, Science 281, 1305-1308.

26. Evan, G. and Littlewood, T. (1998) A matter of life and cell death. Science 281, $1317-1322$.

27. Green, D. R. and Reed, J. C. (1998) Mitochondria and apoptosis. Science 281, 1309-1312.

28. Galle, P. R., Hofmann, W. J., Walczak, H., et al. (1995) Involvement of the CD95 (APO-1/Fas) receptor and ligand in liver damage. J. Exp. Med. 182, 1223-1230.

29. Strand, S., Hofmann, W. J., Grambihler, A., et al. (1998) Hepatic failure and liver cell damage in acute Wilson's disease involve CD95 (APO-1/Fas) mediated apoptosis. Nat. Med. 4, 588-593.

30. Kuhnel, F., Zender, L., Paul, Y., et al. (2000) NFkappaB mediates apoptosis through transcriptional activation of Fas (CD95) in adenoviral hepatitis. J. Biol. Chem. 275, 6421-6427.

31. Faubion, W. A., Guicciardi, M. E., Miyoshi, H., et al. (1999) Toxic bile salts induce rodent hepatocyte apoptosis via direct activation of Fas. J. Clin. Invest. 103, 137-145.

32. Feldstein, A. E., Canbay, A., Angulo, P., et al. (2003) Hepatocyte apoptosis and fas expression are prominent features of human nonalcoholic steatohepatitis. Gastroenterology 125, 437-443.

33. Yin, X. M. and Ding, W. X. (2003) Death receptor activation-induced hepatocyte apoptosis and liver injury. Curr. Mol. Med. 3, 491-508.

34. Zender, L., Hutker, S., Liedtke, C., et al. (2003) Caspase 8 small interfering RNA prevents acute liver failure in mice. Proc. Natl. Acad. Sci. USA 100, 7797-7802.

35. Zhang, H., Cook, J., Nickel, J., et al. (2000) Reduction of liver Fas expression by an antisense oligonucleotide protects mice from fulminant hepatitis. Nat. Biotechnol. 18, 862-867.

36. Mundt, B., Kuhnel, F., Zender, L., et al. (2003) Involvement of TRAIL and its receptors in viral hepatitis. FASEB J. 17, 94-96.

37. Streetz, K. L., Luedde, T., Manns, M. P., and Trautwein, C. (2000) Interleukin 6 and liver regeneration. Gut 47, 309-312.

38. Seglen, P. O., Schwarze, P. E., and Saeter, G. (1986) Changes in cellular ploidy and autophagic responsiveness during rat liver carcinogenesis. Toxicol. Pathol. 14, $342-348$. 